\section{Therapeutische Konzepte bei der Amyotrophen Lateral Sklerose (ALS)}

\author{
A. C. Ludolph ${ }^{1}$ \\ ${ }^{1}$ Klinik für Neurologie, Universitätsklinikum Ulm
}

Therapieansätze bei der ALS beziehen sich einerseits auf pharmakologische Vorgehensweisen, andererseits auf Ansätze der symptomatischen Therapie.

Pharmakologisch hat der Neurologe derzeit nur das Medikament mit dem Wirkstoff Riluzol in der Hand. Riluzol ist hinsichtlich seines Nebenwirkungsprofils sehr gut verträglich und seine Bedeutung wird unterschätzt. Es ist immerhin das einzige Medikament, von dem in vier unabhängigen Studien eine Verlangsamung des Krankheitsverlaufes gezeigt werden konnte. In der ersten Studie, in die Patienten eingeschlossen wurden, die durchschnittlich noch ein Jahr zu leben hatten, gab es einen Überlebensvorteil von knapp vier Monaten. Alle später generierten Daten (retrospektiv und prospektiv) haben gezeigt, dass der Effekt vom Zeitpunkt des Beginns der Therapie abhängig ist. Interessant ist, dass Riluzol zur Klasse der Benzothiazole gehört, einer Gruppe von Substanzen, zu denen auch das Dexpramipexol zählt.

Wahrscheinlich sind symptomatische Ansätze zur Therapie der ALS wirkungsvoller als das Riluzol. Hinsichtlich der Ernährung des Schluckgestörten, aber auch intrinsisch kataboler Patienten kann gesagt werden, dass der BMI ein eindeutiger prognostischer Faktor für diese Erkrankung ist; dabei haben diejenigen Patienten mit einem hohen BMI einen deutlichen Überlebensvorteil gegenüber den Patienten mit einem niedrigen BMI. Therapeutisch relevante Faktoren sind einerseits die Dysphagie, aber auch andererseits ein intrinsischer Katabolismus, der nach der heutigen Erkenntnislage durch eine Beteiligung des Hypothalamus mit bestimmt wird. Es gibt auch Hinweise dafür, dass der LDL-Spiegel, der Cholesterolund der Triglyzerid-Spiegel im Blut ebenfalls positive prognostische Faktoren darstellen. Es darf aber nicht darauf geschlossen werden, dass eine Intervention, die das Ziel hat, hohe Lipidspiegel zu erreichen, von Nutzen ist; hierzu bedarf es einer prospektiven kontrollierten Studie. Der Nutzen der nicht-invasiven Heimbeatmung ist hinsichtlich Lebensqualität und Lebenserwartung gesichert. Das Ausmaß dieses Nutzens hängt von der Form der ALS ab; am meisten profitieren Patient mit thorakalem Beginn; spinale Patienten überleben nach dem Eintritt der durch die $\mathrm{CO}_{2}$-Narkose verursachten
Symptome im Durchschnitt knapp ein Jahr mit der NIV. Um dieses Jahr zu erreichen, muss der bulbäre Patient meist mit einer Maske versorgt werden, die das gesamte Gesicht umfasst.

Es darf nicht unterschätzt werden, welche Rolle eine gute Hilfsmittelversorgung für die Lebensqualität und auch den Aktionsradius des Patienten spielt. Die erforderlichen Hilfsmittel müssen vorausschauend verordnet werden; zu den wichtigen ALS-spezifischen Hilfsmitteln gehören ein Rollstuhl, auch ein elektrisch betriebener Rollstuhl, Kommunikationshilfen, aber auch konventionelle Hilfsmittel wie eine Peroneusschiene oder eine Nackenstütze.

Ein wichtiges Thema ist auch die Diskussion nicht belegter Therapien. Auf dem Feld der ALS tummeln sich unseriöse Kolleginnen und Kollegen, die mit Hilfe von Akupunktur, osteopathischen Methoden aber auch der immer präsenten „Stammzelltherapie“ eine Gefahr für den Patienten darstellen. Es ist eine vornehme ärztliche Aufgabe, den Patienten über die Motive dieser Therapeuten vollständig aufzuklären. Derzeitige Therapiestudien zielen einerseits auf die Beeinflussung der Ätiologie, andererseits auf die Beeinflussung der Pathogenese der Erkrankung. Ätiologisch orientiert ist der Einsatz von Oligonukleotiden, die die Transkription spezifischer Gene verhindern sollen. Diese Methoden werden zurzeit für die Superoxid Dismutase (SOD1) und das Gen C9ORF72 entwickelt. Eine aktuelle Studie beschäftigt sich mit der Translation der SOD1; das Malariamedikament Pyrimethamin reduziert die Expression der toxischen SOD1. Andere Therapiestudien übernehmen Ansätze aus dem Parkinsonfeld (Rasagilin), wieder andere übersetzen Befunde, die zeigen, dass das Protein Nogo das Sprouten im Muskel verhindert, durch den Einsatz monoklonaler Antikörper gegen dieses Protein. Weitere Studien befassen sich unspezifischer mit einer Erhöhung der Kontraktilität des Muskels.

Aus meiner Sicht kann man in der Zukunft therapeutische Fortschritte auf pharmakologischer Ebene von Hypothesengestützten Ansätzen erwarten (Modifier Gene wie PGC1 alpha, Ephrin A4 Rezeptor), aber auch von den neuen Erkenntnissen, die den Ausbreitungsmodus molekularer Markermoleküle (TDP-43) kennzeichnen.

Interessenkonflikt: Es bestehen keinerlei Interessenkonflikte.

\footnotetext{
Bibliografie

Dol http://dx.doi.org/10.1055/s-0033-1346716

Drug Res 2013; 63, Suppl. 1: S21-S21

(c) Georg Thieme Verlag KG Stuttgart · New York .

ISSN 2194-9379
} 